\title{
Modelling koala density using incidental koala sightings in South East Queensland, Australia (1997-2013)
}

\author{
Ravi Dissanayake ${ }^{1}$, Emanuele Giorgi ${ }^{2}$, Mark Stevenson ${ }^{3}$, Rachel Allavena ${ }^{4}$, and Joerg \\ Henning $^{4}$ \\ ${ }^{1}$ The University of Queensland \\ ${ }^{2}$ Lancaster University Lancaster Medical School \\ ${ }^{3}$ University of Melbourne Faculty of Veterinary and Agricultural Sciences \\ ${ }^{4}$ The University of Queensland Faculty of Natural Resources Agriculture and Veterinary \\ Science
}

October 1, 2020

\begin{abstract}
The koala, Phascolarctos cinereus, is an iconic Australian wildlife species, but faces rapid decline in South-East Queensland (SEQLD). For conservation planning, estimating koala populations is crucial. Systematic surveys are the most common approach to estimate koala populations, but such surveys are restricted to small geographic areas, they are costly and conducted infrequently. Public interest and participation in the collection of koala sightings is increasing in popularity, but such data is generally not used for population estimation. We used incidental sightings of koalas reported by members of the public from 1997-2013 in SEQLD to estimate the yearly spatio-temporal koala sightings density. For this, a spatio-temporal point process model was developed accounting for observed koala density, spatio-temporal detection bias and clustering. The density of koalas varied throughout the study period due to the heterogeneous nature of koala habitat in SEQLD, with density estimates ranging between 0.005 to 8.9 koalas per km2. The percentage of land areas with very low sightings densities (0-0.25 koalas per km2) remained similar throughout the study period representing in average (SD) 68.3\% (0.06) of the total study area. However, land areas with more koalas per $\mathrm{km} 2$ showed larger annual variations, with koala mean (SD) densities of 0.25-0.5, 0.5-1, 1-2, 2-5 and $>5$ koalas per km2 representing $16.8 \%(0.21), 13.8 \%(0.25), 0.7 \%(0.20), 0.3 \%(0.13)$, and $0.2 \%(0.1)$ of the study area in South-East Queensland, respectively.We did find that clustering of koala sightings was not prominently different between the mating and non-mating seasons of koalas. While acknowledging the limitations associated sightings data, we developed a statistical model that addressed the spatio-temporal bias associated with observed koala sightings and provided long-term relative koala density estimates for one of the largest koala populations of Australia.
\end{abstract}

\begin{abstract}
The koala, Phascolarctos cinereus, is an iconic Australian wildlife species, but faces rapid decline in SouthEast Queensland (SEQLD). For conservation planning, estimating koala populations is crucial. Systematic surveys are the most common approach to estimate koala populations, but such surveys are restricted to small geographic areas, they are costly and conducted infrequently. Public interest and participation in the collection of koala sightings is increasing in popularity, but such data is generally not used for population estimation. We used incidental sightings of koalas reported by members of the public from 1997-2013 in SEQLD to estimate the yearly spatio-temporal koala sightings density. For this, a spatio-temporal point process model was developed accounting for observed koala density, spatio-temporal detection bias and clustering. The density of koalas varied throughout the study period due to the heterogeneous nature of koala habitat in SEQLD, with density estimates ranging between 0.005 to 8.9 koalas per $\mathrm{km}^{2}$.
\end{abstract}


The percentage of land areas with very low sightings densities (0-0.25 koalas per $\left.\mathrm{km}^{2}\right)$ remained similar throughout the study period representing in average (SD) $68.3 \%$ (0.06) of the total study area. However, land areas with more koalas per $\mathrm{km}^{2}$ showed larger annual variations, with koala mean (SD) densities of $0.25-0.5,0.5-1,1-2,2-5$ and $>5$ koalas per $\mathrm{km}^{2}$ representing $16.8 \%(0.21), 13.8 \%(0.25), 0.7 \%(0.20), 0.3 \%$ $(0.13)$, and $0.2 \%(0.1)$ of the study area in South-East Queensland, respectively.

We did find that clustering of koala sightings was not prominently different between the mating and nonmating seasons of koalas. While acknowledging the limitations associated sightings data, we developed a statistical model that addressed the spatio-temporal bias associated with observed koala sightings and provided long-term relative koala density estimates for one of the largest koala populations of Australia. In future research, the proposed model proposed here could be used for systematic survey data and ultimately for combining koala survey data with koala sightings data and remove the spatial bias more reliably.

Key words: Koala, modelling, population, citizen science, Queensland

\section{Introduction}

Direct observations and counting of koalas in the field faces many challenges, because koalas are difficult to detect in their natural habitat and they are widely dispersed (Ellis, et al. 2013, Masters, et al. 2004, McGregor, et al. 2013). Various methods are used to count koalas and estimate koala population density: systematic field surveys (David S. Dique, et al. 2003), distance sampling (D. S. Dique, et al. 2003), counting the number of faecal pellets under trees (Seabrook, et al. 2011, Sullivan, et al. 2002, Sullivan, et al. 2003), capture-mark-recapture methods (Masters, Duka, Berris and Moss 2004) and community surveys (Hollow 2015). Counts of vocalisations heard and spotlight surveys are also sometimes used (Smith and Andrews 1997).

Systematic surveys of strip transects (Dique, et al. 2004) and distance sampling using line transects are the most common methods to estimate koala density, but distance sampling techniques are only suitable for small areas because they are labour intensive and therefore expensive (Kjeldsen, et al. 2015). Research has described the relationship between koala's tree preference and the presence of koala scat (Ellis, FitzGibbon, Melzer, Wilson, Johnston, Bercovitch, Dique and Carrick 2013) and koala scat prevalence has been shown to correlate well with koala density (Ellis, et al. 1998, Lunney, et al. 2009). Scat surveys are less expensive than systematic surveys and have been therefore been used to estimate koala population density (McAlpine, et al. 2006, Rhodes, et al. 2008). Postal surveys of targeted communities and incidental sightings of koalas by members of the public have also been used to estimate population counts (Cork, et al. 2000, Lunney, Crowther, Shannon and Bryant 2009, Lunney, et al. 2016, Predavec, et al. 2016, Sequeira, et al. 2014). These methods are suitable for smaller geographical areas with varying success and require statistical analysis to estimate population counts based on reported koala numbers (Santika, et al. 2014).

To review koala density estimates for conservation purposes, it is important to generate long-term datasets of koala populations over large geographical areas rather than to generate population counts at infrequent intervals (Ellis, Sullivan, Lisle and Carrick 1998, Lunney, et al. 2014, Lunney, Predavec, Miller, Shannon, Fisher, Moon, Matthews, Turbill and Rhodes 2016). Long-term survey data have been used to predict koala populations. A recent study in South-East Queensland (Santika, et al. 2015) attempted to estimate the geographic distribution of koala populations across a wide geographical area using spatial modelling techniques informed by long term (Rhodes, et al. 2015) line and strip transect survey data (1996 and 2015) collected by distance sampling. Ecological modelling techniques can also provide an alternative to active, longitudinal data collection (Schmolke, et al. 2010), although their validity is questionable in the absence of standardised methods for estimating wildlife density and distribution (Dique, Preece, Thompson and Villiers 2004, McGregor, Kerr and Krockenberger 2013).

With the advancement of communication technologies and the widespread availability of dedicated mobile applications, public participation in the collection of wildlife data is increasing in popularity. Attempts have been made to estimate wildlife populations using incidental sighting data alone, and/or in combination with 
survey data (Dorazio 2014, Sequeira, Roetman, Daniels, Baker and Bradshaw 2014). For instance, member of the public were invited to take part in collecting data on koala sightings as part of a program titled the 'Great Koala Count' in the Australian states of New South Wales and South Australia in 2012 (Sequeira, Roetman, Daniels, Baker and Bradshaw 2014). The 'Great Koala Count' has generated large number of incidental koala sighting using specific guidelines for data collection in pre-identified geographical areas in those two states. In South-East Queensland incidental koala sightings are collected since 1997, although no formal field protocols are provided to members of the public for the data collection (Dissanayake, et al. 2019). While these data have been used to describe koala population trends and to describe spatial biases identified (Dissanayake, Stevenson, Allavena and Henning 2019), it has not yet been used to estimate long-term koala density. In this study, we have developed a modelling approach to estimate koala density from observed sightings data over a period of 17 years, while addressing spatio-temporal detection biases and potentially clustering of observations.

\section{Materials and methods}

\section{Study area}

The area of interest for this study comprised 15 local government areas (LGAs) located in South-East Queensland (Figure 1). South-East Queensland has a higher population of koalas compared with other areas of Queensland (Dique, Preece, Thompson and Villiers 2004), but has also a high urban development. The point locations of koala sighting events (details provided below) were plotted on a map of South-East Queensland, indicating relatively few sightings in the North and to the far West of Queensland. To develop an observation window that was tractable for modelling we constructed a convex hull around all koala sighting locations and then dilated the convex hull by 3 kilometers to accommodate the home range of koalas sighted on the border of the observation window (de Oliveira, Murray, de Villiers, \& Baxter, 2014). Refinement of the study area in this way reduced the number of constituent LGAs from 15 to 11 and included parts of 3 remaining LGAs. The selected LGAs were in the Eastern and Central part of South-East Queensland. The northern LGA of the Fraser Coast was excluded due to an absence of koala sighting records. This reduced the study from 57,800 square kilometers to 30,500 square kilometers.

Figure 1. Local Government Areas (LGA) of South-East Queensland, Australia (N=15). The LGAs for which koala population densities were calculated are shown in the rectangle (Green areas represents the main koala conservation in South-East Queensland).

\section{Koala sighting data}

Incidental koala sightings reported in South-East Queensland between 1997 and $2013(N=14,250$ sightings) were retrieved from KoalaBASE, a database of clinical admission and sightings data of koalas, which developed by the University of Queensland's School of Veterinary Science (www.koalabase.com.au). The dataset comprised of the date of the sighting as well as the longitude and latitude of the location, where the koala was observed. We assumed that if two koalas were reported in the same area in the same month, these could be repeated sightings of the same animal. The term 'area' in this context represents the estimated home range for a koala, which was assumed to be circular with an average size of $0.35 \mathrm{~km}^{2}$ (de Oliveira, et al. 2014). Thus, the minimum distance between two koalas was given byd $=2 \times \sqrt{A \div \pi}$, or 666 metres. Figure 2a shows a selection of koala sighting locations for month 1, which are minimum of 666 metres apart and excluded other sightings for the month, while Figure $2 \mathrm{~b}$ shows a selection of koala sightings for month 2. Because home ranges of koalas can overlap (Ellis, et al. 2009), Figure 2c shows the two monthly data sets combined. In Figure 2c sightings are permitted to be closer than the minimum distance set for individual monthly observations, acknowledging that koala home ranges overlap here. Following this approach, monthly sightings observations for each year over the 17 years period were compiled, resulting in a total of 17 years $\times 12$ months $=204$ selections. By adopting this approach, we permitted a koala (i.e. the same koala or a different koala) to be seen in the same area at a maximum of 12 times per year. 
Figure 2. Illustration of the method used to select incidental koala sightings to estimate koala sightings density. Circles represent an average home range area for a koala. Figure 2a shows a selection of koala sighting locations for month 1 , while Figure $2 \mathrm{~b}$ shows a selection of koala sightings for month 2 . Figure $2 \mathrm{c}$ shows the two monthly data sets combined. Following this approach, monthly sightings observations for each year over the 17 years period were compiled and used a subset of sightings to estimate koala sightings density.

\section{Covariates}

The following spatial covariates were used as explanatory variables in the model:

- Distance to primary roads (meters)

- Land lot density (number of lots per square kilometre)

- Mean temperature of the hottest month (centigrade)

- Mean temperature of the coldest month (centigrade)

- Precipitation of the driest month (millimetres)

- Precipitation of the wettest month (millimetres)

- Mean elevation from the sea level (meters)

- Foliage projective cover (proportion)

The covariate Distance to primary roads was considered to only influence spatio-temporal bias associated with these sightings (Dissanayake, Stevenson, Allavena and Henning 2019), since it quantifies the easiness of access to koala habitat by an observer. The other covariates can be assumed to mostly influence observed koala sightings density. These covariates were obtained from online spatial databases (http://qldspatial.information.qld.gov.au, http://www.bom.gov.au) and included as raster maps with a 1 square kilometre resolution.

\section{Spatio-temporal modelling of koala sighting density}

The koala sightings were modelled as a realisation of a spatio-temporal point process model (STPP) model (Baddeley, et al. 2016). STPPs are a useful statistical tool that allow to model the spatial and temporal variation of sightings within a region and time window of interest. Within this modelling framework two main methods can be distinguished: (1) mechanistic models, where subject matter knowledge is used to inform the probability that a sighting will occur at a particular location and a particular time point; and (2) empirical models, where the objective is to use observational data to inform estimates of koala density. The approach used here is a combination of the two methods.

More specifically, we assumed that the koala density at a particular location and a particular time is dependent on the three groups of variables: (a) variables that relate to the spatio-temporal detection bias; (b) spatio-temporally referenced variables that are known to be associated with koala density; and (c) variables that reflect well-established knowledge on the home range of koalas. The main mechanistic component of the model is the third group of variables, while the first two are modelled as a log-linear regression of koala density. A partial likelihood approach was used to fit the model (Diggle, et al. 2010).

Three factors were considered in the spatio-temporal modelling of koala density: spatio-temporal detection bias $b(x, t)$, observed koala density $q(x, t)$, and clustering $\mathrm{r}\left(\mathrm{x}, \mathrm{t} \mid \mathrm{H}_{\mathrm{t}}\right)$. Let $\mathcal{H}_{t}$ denote the full story of the process up to yeart. We assumed that conditionally on $\mathcal{H}_{t}$, the intensity $\lambda$ of the process is at a location $x$ in year $t$ was given by:

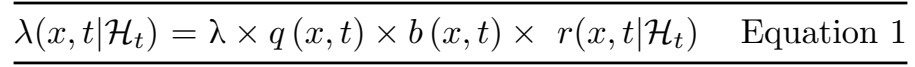

The observed koala density $q(x, t)$ and the spatio-temporal detection bias $b(x, t)$ were modelled as a loglinear regression on covariates $d(x, t)$ and $e(x, t)$.

$$
\overline{\log \{b(x, t)\}=\beta^{\top} d(x, t) \quad \text { Equation } 2}
$$




$$
\overline{\log \{q(x, t)\}=\gamma^{\top} e(x, t) ; \quad \text { Equation } 3}
$$

In this analysis, we used "Distance to primary roads" as $d(x, t)$ and used all the other covariates as $e(x, t)$. The third factor correspond to clustering of koalas that cannot be explained by $d(x, t)$ and and $e(x, t)$, were modelled as: with

\begin{tabular}{|c|c|}
\hline$\left.r\left(x, t \mid \mathcal{H}_{t}\right)=\prod_{j: t_{i}} 1+[\theta(t)-1] f\left(\left\|x-x_{j}\right\|\right)\right\}$ & Equation 4 \\
\hline $\begin{array}{l}f\left(\| x-x_{j}\right)= \\
\left\{\begin{aligned} & \exp \left\{-\left\|x-x_{j}\right\| / \phi\right. \text { if }\left\|x-x_{j}\right\| \quad \text { \&amp } ;<u(x) \\
& \text { if }\left\|x-x_{\mathrm{j}}\right\|, \quad \text { \&amp; } \geq u(x)\end{aligned}\right.\end{array}$ & Equation 5 \\
\hline
\end{tabular}

and $u(x)$ is the square root of the koala home range (in $\mathrm{km}^{2}$ ) at location $x$. In Equation 4 the parameter $\theta(t)$ regulates the strength and direction of the spatial interaction between sightings: $0<\theta(t)<1$ corresponds to an inhibitory point-process; $\theta(t)=1$ is the case of no interaction; and $\theta(t)>1$ represents the situation where koala sightings are spatially aggregated. We let $\theta(t)$ vary between the mating and non-mating seasons as we expected a stronger spatial interaction of koalas and thereby potential aggregations of sightings during the former period. This seasonality was included as binary variable with 1 being assigned the for mating season $\theta 2$ (August to September (de Oliveira, Murray, de Villiers and Baxter 2014)) and 0 representing the non-mating season $\theta 1$ for rest of the year We fit the model using partial likelihood (Lp) given by to estimate koala sightings density for the observation period of 17 years:

$$
\begin{array}{lll}
\hline L=\sum_{i=1}^{n} \log \frac{q\left(x, t_{i}\right) \mathrm{b}\left(x_{i},\right.}{\int_{\dot{A}} b\left(x, t_{i}\right) q\left(x, t_{i}\right) r\left(x, t \mid \mathcal{H}_{\mathrm{ti}}\right) d x} & \mathrm{r}\left(x, t_{i} \mathcal{H}_{\mathrm{ti}}\right. & \text { Equation } 6 \\
\hline
\end{array}
$$

In this model, the term $\theta$ parameterises the strength and the direction of the interaction between koalas. A value of $\theta<1$ means repulsion, $\theta=1$ means no interaction and $\theta>1$ means aggregation. The term $\varphi$ provides a measure of the spatial scale of interaction. If $\theta>1$ and $\varphi$ is large then koalas aggregate in large areas. When $\theta>1$ and $\varphi$ is small koalas aggregate in smaller areas.

The likelihood function given by the above equation was maximized using a numerical optimization procedure implemented in the "nlimnb" function in the R (version 3.5.1) software environment. Since the model specified was not available in any of the existing $\mathrm{R}$ packages, the algorithm used in this analysis was developed in $\mathrm{R}$.

\section{Results}

The selected monthly sightings over the 17-year observation period were merged into a single data set, reducing the number of sightings from 14,256 to 6,580. The total number of sightings and the subset of sightings used for the data analysis are shown in Figures 3a and 3b.

Figure 3 . Maps of the study areas in South-East Queensland, Australia showing: (a) point location of all incidental koala sightings recorded in KoalaBASE for the period January 1997 to December 2013 ( $\mathrm{n}=$ 14,256); and (b) the subset of koala sightings $(\mathrm{n}=6,580)$ using the data analysis to estimate koala density.

A line plot showing temporal pattern of the koala population by calendar time estimated from the spatiotemporal point process model using koala sightings $(n=6,580)$ is shown in Figure 4 . The koala population was low for the period 1997 to 2000 without prominent peaks, then fluctuated with peaks from 2001 to 2008 (with biennial larger peaks), before reaching large seasonal peaks in 2009 and 2010, declining again to peaks similar to pre-2009 period, followed by another large peak in 2013. 
Figure 4. Koala population by calendar time estimated from the spatio-temporal point process model using koala sightings $(\mathrm{n}=6,580)$ recorded in South-East Queensland, 1997-2013.

Parameter estimates from a spatio-temporal point process model used to estimate koala population densities in South-East Queensland between 1997 and 2013 are shown in Figure 1. The coefficients of land lot density and mean temperature of the coldest month had positive coefficients, while the coefficients of the other covariates included in the model were negative (Table 1).

Table 1. Estimated parameters from a spatio-temporal point process model used to estimate koala population densities in South-East Queensland between 1997 and 2013.

The relative estimated koala population density in South-East Queensland, 1997-2013 is shown in Figure 5 ranging from 0 to 6 or above koalas per $\mathrm{km}^{2}$, with the spatial distribution of sightings remaining more or less the same. Based on the model presented in Equation 6,? was $>0$ and $\varphi$ was small suggesting that koalas aggregate in large areas. The estimates are consistent with the observed sightings, with no koalas or very low koala density in the western part of the study area and increasing density towards the Eastern coast of South-East Queensland, with prominent pockets of high koala density in known areas with good koala habitat.

Figure 5. Estimated relative koala population density (koalas per $\mathrm{km}^{2}$ ) in South-East Queensland, 19972013. Estimates were derived from a spatio-temporal point process model using koala sightings data $(\mathrm{n}=$ $6,580)$.

The percentage of land area in South-East Queensland with varying koala sightings density (koalas per $\mathrm{km}^{2}$ ) for each year of the 1997-2013 period is shown in Table 2. The percentage of land areas with very low sightings densities (0-0.25 koalas per $\left.\mathrm{km}^{2}\right)$ remained similar throughout the study period representing in average (SD) $68.3 \%$ (0.06) of the total study area (Table 2). However, land areas with more koalas per $\mathrm{km}^{2}$ showed larger variations over the years, with koala mean (SD) densities of $0.25-0.5,0.5-1,1-2,2-5$ and > 5 koalas per $\mathrm{km}^{2}$ representing $16.8 \%(0.21), 13.8 \%(0.25), 0.7 \%(0.20), 0.3 \%(0.13)$, and $0.2 \%(0.1)$ of the study area in South-East Queensland, respectively.

Table 2. Percentage of land area in South-East Queensland, 1997-2013, with varying koala population density (koalas per $\mathrm{km}^{2}$ ). Estimates were derived from a spatio-temporal point process model using koala sightings data $(\mathrm{n}=6,580)$.

\section{Discussion}

We present here the results of spatio-temporal point process model, where relative koala population density was estimated considering spatio-temporal detection bias, observed koala densities and potential clustering effects. As partial likelihood estimation was used, the intercept was not calculated and absolute koala sightings density could not be estimated. However, relative koala population density estimates were produced for each year of the 17-year observation period.

The density of the koala population in South-East Queensland varied throughout the study region due to the heterogeneous nature of koala habitat, with density estimates ranging from 0.005 to 8.9 koalas per $\mathrm{km}^{2}$. Limited information of koala densities exist in Australia, but Rhodes, Beyer, Preece and McAlpine (2015) estimated koala densities varying between 0.001 and 11.0 koalas per ha in coastal regions of South-East Queensland, with an average of 0.04 koalas per ha (or 4 koalas per $\mathrm{km}^{2}$ ). However, the model developed by Rhodes et al. (2015) utilized data collected through multiple systematic surveys, which were implemented in small areas and did not predict koala populations across large geographic areas due to uncertainties associated with extrapolations. In fact, extrapolating koala densities from statistical models for large geographical areas is questionable as koala habitat is not continuously distributed. To avoid this fallacy, densities should be predicted to strata of different habitat types (Dique, Preece, Thompson and Villiers 2004).

Actual koala numbers are very difficult to estimate. In 2010, the Department of Environmental Heritage 
and Protection (DEHP) predicted that the Queensland koala's population was between 157,000 and 177,000 animals, while the Threatened Species Scientific Committee of Australia estimated Queensland's koala population to be approximately 167,000 animals in 2010, representing as 43\% decline from 1990 (Rhodes, Beyer, Preece and McAlpine 2015). Another study estimated Queensland's koala population to be about 79,300 in 2012 (Adams-Hosking, et al. 2016). Using expert elicitation methods the koala population for the whole of Australia was approximated to be 329,000 individuals (ranging from 144,000 to 605,000) (Adams-Hosking, McBride, Baxter, Burgman, de Villiers, Kavanagh, Lawler, Lunney, Melzer, Menkhorst, Molsher, Moore, Phalen, Rhodes, Todd, Whisson, McAlpine and Richardson 2016).

The results of the statistical model presented here provide estimates of yearly koala population densities, which are informed and therefore strongly influenced by observed sightings. Our model results showed increased koala population densities in some years, which might simply represent a higher observed fraction of koalas from the true koala population. We could also show strong clustering of koalas in locations in and around the Moreton Bay and Redland areas which is similar to the high density areas identified by Rhodes, Beyer, Preece and McAlpine (2015) using systematic field survey data. However, our model did identify low densities of koalas in the western part of South-East Queensland whereas Rhodes et al. (2015) predicted higher densities there, although this was probably due to the uncertainty associated with the model estimates for this region.

Importantly, we were able to estimate koala population density over time and space while incorporating a range of covariates expected to be associated with observed sighting densities or spatio-temporal detection bias. For example, distance to primary roads was considered to be covariate predominately influencing spatio-temporal bias, while foliage protective cover was influencing an observer's ability to sight a koala and therefore impacting on observed sighting density. However, the contribution of covariates to the two different components of the model cannot be quantified as these components were included as additive factors on a log-scale in the model. Considering that covariates with a negative sign, would decrease estimated koala population density, our model indicated that larger distance to primary roads, denser foliage, higher altitude, but also increases precipitation would result in less sightings being reported. In contrast, increased lot density and warmer temperatures in the colder months were associated with increases population densities.

Uncertainties in estimated koala densities can be further reduced if additional data are collected at the time of each sighting event. This data could then be used in the modelling approaches to estimate and remove the effect of the bias on population density estimates. In our study, no observer-related variables were collected at the time when koalas were sighted. It has been shown that the probability of detection of a koala by an observer varies with previous experience of detecting koalas: an experienced observer can have a detection rate of around $70 \%$, while an inexperienced observer might have a detection rate of only $30 \%$ (Corcoran, et al. 2019). As a result, many koalas may go undetected simply because of the lack of observer experience. The situation is somewhat different in systematically conducted field surveys by trained individuals where detection probabilities are estimated (Rhodes, Beyer, Preece and McAlpine 2015). Thus, incidental sightings reported by members of the public represent a biased sample of the koala population at any given time, but the collection of data on experience of observers at the time of the sighting, could provide value information to address this bias.

Koala sightings vary between seasons of the year. Such seasonal variations might be due to more frequent dispersal of koalas during breeding periods, but also due to better visibility of animals and better weather conditions that are more favorable for people to go outdoors and spot koalas. Interestingly, the results of our model indicated that clustering of koalas is not prominently different between the mating (theta $1=$ 2.0056 ) and non-mating seasons of koalas (theta $2=2.029$ ). This might be explained by koalas being solitary animals and although they travel over larger distances in the breeding season, their greater mobility might not necessary be associated with clustering of animals.

We included an average home range of koalas in our model, as we did not have detailed koala home range information for different parts of our study area. We realize that koala home ranges are not uniform and even within the Redland Local Government Area, koala home ranges of koalas vary between 0.05 and 0.55 
$\mathrm{km}^{2}$ (de Oliveira, Murray, de Villiers and Baxter 2014). The precisions of koala densities could be improved, if home ranges appropriate for each habitat types are included in the model.

It has been predicted that drier and warmer climatic conditions have an undesirable impact on koala habitat and thereby negatively impacting koala populations (Adams-Hosking, et al. 2011). Unfortunately, our study was constrained by the non-availability of temporally varying covariates. As a result, the temporal effect of covariates, such as the impact of temperature changes over time on koala densities, could not be quantified.

Overall, while acknowledging the limitations associated sightings data collected by members of the public, we developed a statistical model that addressed the spatio-temporal bias associated with observed koala sightings and provided long-term relative koala density estimates for one of the largest koala populations of Australia over a 17-year period. In future research, the model proposed here could be used for systematic survey data and ultimately for combining (spatially restricted, but more precise) koala survey data with koala sightings data, that is incidental and often biased by nature, but often collected over large geographical areas.

\section{Acknowledgements}

We thank Department of Environment and Science (DES), Queensland for providing access to the sightings data as part of the KoalaBASE project. We also thank the staff of the Moggill Koala Rehabilitation Centre at Brisbane, for providing insights into the data collection.

\section{Author Contributions}

R.B.D. performed data processing, R.B.D and E.G analysed data, and R.B.D and E.G wrote the manuscript. R.B.D., E.G., J.H., designed the study. J.H., M.S. and R.A. provided input in the manuscript writing. All authors reviewed the manuscript.

Competing Interests: The authors declare no competing interests.

\section{Data Availability}

The incidental koala sighting dataset analyzed in this study is available at www.koalabase.com.au.

\section{References}

Adams-Hosking, C., et al. 2011. Modelling climate-change-induced shifts in the distribution of the koala. Wildlife Research 38: 122 .

Adams-Hosking, C., et al. 2016. Use of expert knowledge to elicit population trends for the koala (Phascolarctos cinereus). - Diversity and Distributions 22: 249-262.

Baddeley, A., et al. 2016. Spatial point patterns : Methodology and applications with R (Interdisciplinary statistics). - Boca Raton, FL: CRC Press, Taylor \& Francis Group.

Corcoran, E., et al. 2019. Automated detection of koalas using low-level aerial surveillance and machine learning. - Sci Rep 9: 3208.

Cork, S. J., et al. 2000. Improving habitat models and their utility in koala conservation. - Conservation Biology 14: 660-668.

de Oliveira, S. M., et al. 2014. Ecology and movement of urban koalas adjacent to linear infrastructure in coastal south-east Queensland. - Australian Mammalogy 36: 45-54.

Diggle, P. J., et al. 2010. Partial-likelihood analysis of spatio-temporal point-process data. - Biometrics 66: $347-54$.

Dique, D. S., et al. 2003. Dispersal patterns in a regional koala population in south-east Queensland. Wildlife Research 30: 281-290.

Dique, D. S., et al. 2003. Evaluation of line-transect sampling for estimating koala abundance in the Pine Rivers Shire, south-east Queensland. - Wildlife Research 30: 127. 
Dique, D. S., et al. 2004. Determining the distribution and abundance of a regional koala population in south-east Queensland for conservation management. - Wildlife Research 31: 109.

Dissanayake, R. B., et al. 2019. The value of long-term citizen science data for monitoring koala populations. - Scientific Reports 9:

Dorazio, R. M. 2014. Accounting for imperfect detection and survey bias in statistical analysis of presenceonly data. - Global Ecology and Biogeography 23: 1472-1484.

Ellis, W., et al. 2013. Koala habitat use and population density: using field data to test the assumptions of ecological models. - Australian Mammalogy 35: 160-165.

Ellis, W. A. H., et al. 1998. The spatial and temporal distribution of koala faecal pellets. - Wildlife Research 25: $663-668$.

Ellis, W. A. H., et al. 2009. Spatiotemporal dynamics of habitat use by koalas: the checkerboard model. Behavioral Ecology and Sociobiology 63: 1181-1188.

Hollow, B. R., P. E. J. Walter, M. Daniels, C. B. 2015. Citizen science for policy development: The case of koala management in South Australia. 47: 126-136.

Kjeldsen, S. R., et al. 2015. Genome-wide SNP loci reveal novel insights into koala (Phascolarctos cinereus) population variability across its range. - Conservation Genetics 17: 337-353.

Lunney, D., et al. 2009. Combining a map-based public survey with an estimation of site occupancy to determine the recent and changing distribution of the koala in New South Wales. - Wildlife Research 36: 262-273.

Lunney, D., et al. 2014. Extinction in Eden: identifying the role of climate change in the decline of the koala in south-eastern NSW. - Wildlife Research 41: 22.

Lunney, D., et al. 2016. Interpreting patterns of population change in koalas from long-term datasets in Coffs Harbour on the north coast of New South Wales. - Australian Mammalogy 38: 29-43.

Masters, P., et al. 2004. Koalas on Kangaroo Island: from introduction to pest status in less than a century. - Wildlife Research 31: 267-272.

McAlpine, C. A., et al. 2006. The importance of forest area and configuration relative to local habitat factors for conserving forest mammals: A case study of koalas in Queensland, Australia. - Biological Conservation 132: $153-165$.

McGregor, D. C., et al. 2013. The distribution and abundance of an island population of Koalas (Phascolarctos cinereus) in the far north of their geographic range. - PLoS One 8: e59713.

Predavec, M., et al. 2016. The contribution of community wisdom to conservation ecology. - Conserv Biol 30: 496-505.

Rhodes, J. R., et al. 2008. Regional variation in habitat-occupancy thresholds: a warning for conservation planning. - Journal of Applied Ecology 45: 549-557.

Rhodes, J. R., et al. 2015. South East Queensland Koala Population Modelling Study. UniQuest, Brisbane, Australia. - In.

Santika, T., et al. 2014. Modelling species distributional shifts across broad spatial extents by linking dynamic occupancy models with public-based surveys. - Diversity and Distributions 20: 786-796.

Santika, T., et al. 2015. Assessing spatio-temporal priorities for species' recovery in broad-scale dynamic landscapes. - Journal of Applied Ecology 52: 832-840.

Schmolke, A., et al. 2010. Ecological models supporting environmental decision making: a strategy for the future. - Trends in Ecology \& Evolution 25: 479-486. 
Seabrook, L., et al. 2011. Drought-driven change in wildlife distribution and numbers: a case study of koalas in south west Queensland. - Wildlife Research 38: 509-524.

Sequeira, A. M. M., et al. 2014. Distribution models for koalas in South Australia using citizen sciencecollected data. - Ecology and Evolution 4: 2103-2114.

Smith, A. P. and Andrews, S. 1997. Koala habitat, abundance and distribution in the Pine Creek study area. Report to State Forests of New South Wales. - In.

Sullivan, B. J., et al. 2002. Low-density koala (Phascolarctos cinereus) populations in the mulgalands of south-west Queensland. I. Faecal pellet sampling protocol. - Wildlife Research 29: 455-462.

Sullivan, B. J., et al. 2003. Low-density koala (Phascolarctos cinereus) populations in the mulgalands of south-west Queensland. III. Broad-scale patterns of habitat use. - Wildlife Research 30: 583-591.
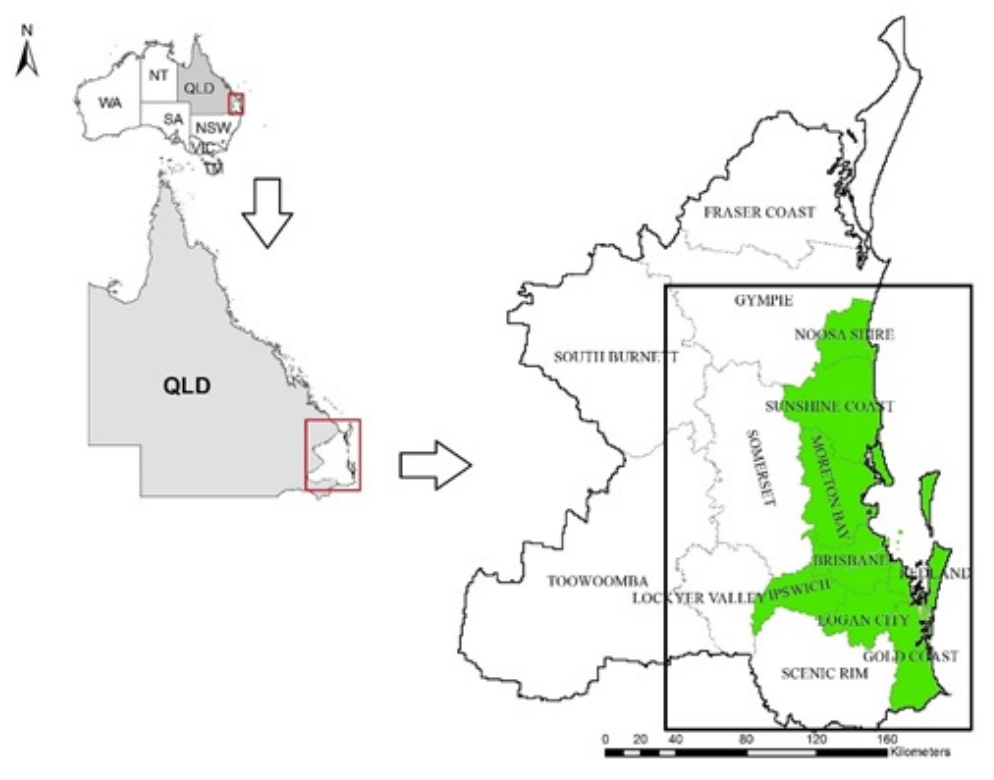

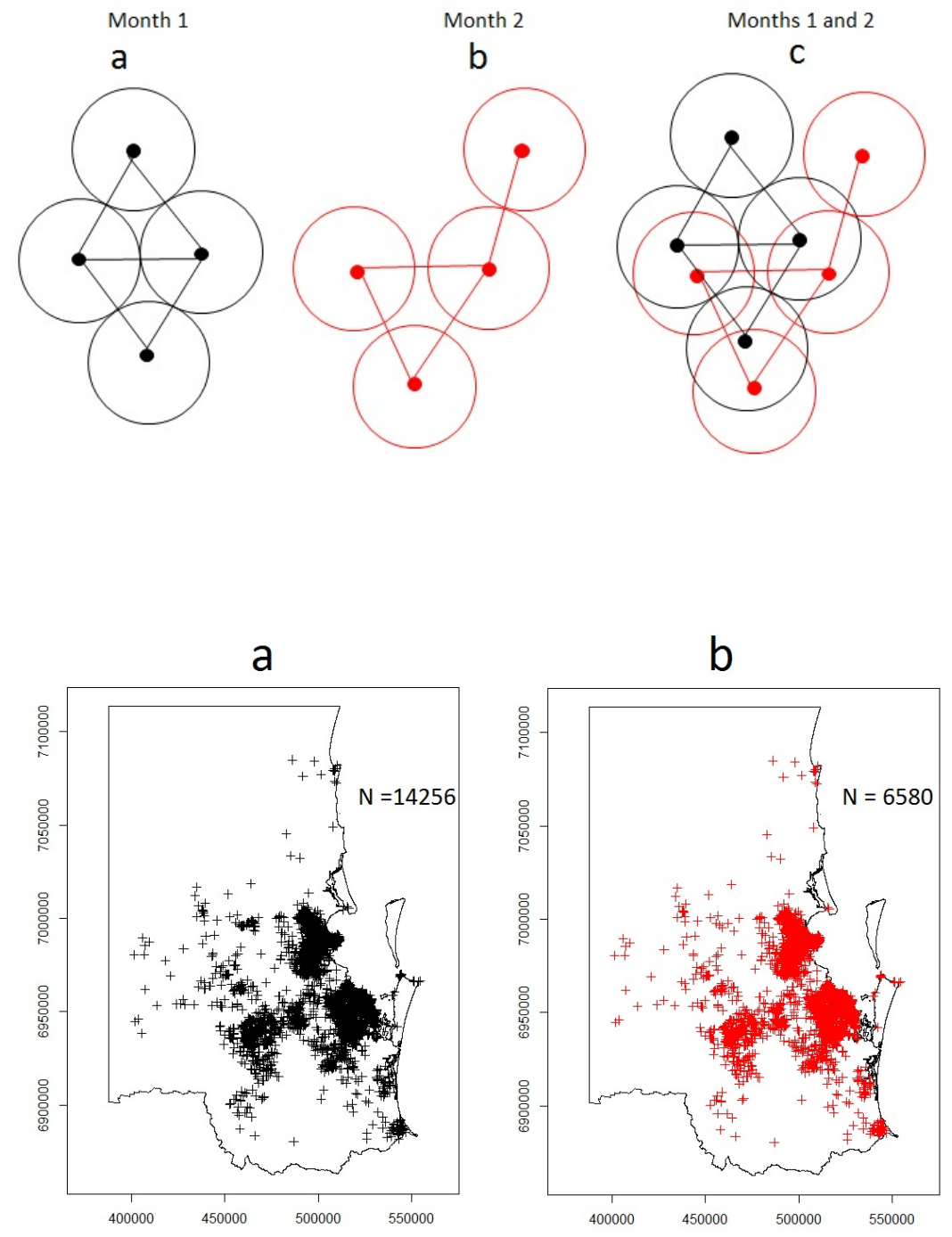

\section{Hosted file}

Table 1.pdf available at https://authorea.com/users/363574/articles/484300-modelling-koaladensity-using-incidental-koala-sightings-in-south-east-queensland-australia-1997-2013

\section{Hosted file}

Table2.pdf available at https://authorea.com/users/363574/articles/484300-modelling-koaladensity-using-incidental-koala-sightings-in-south-east-queensland-australia-1997-2013 


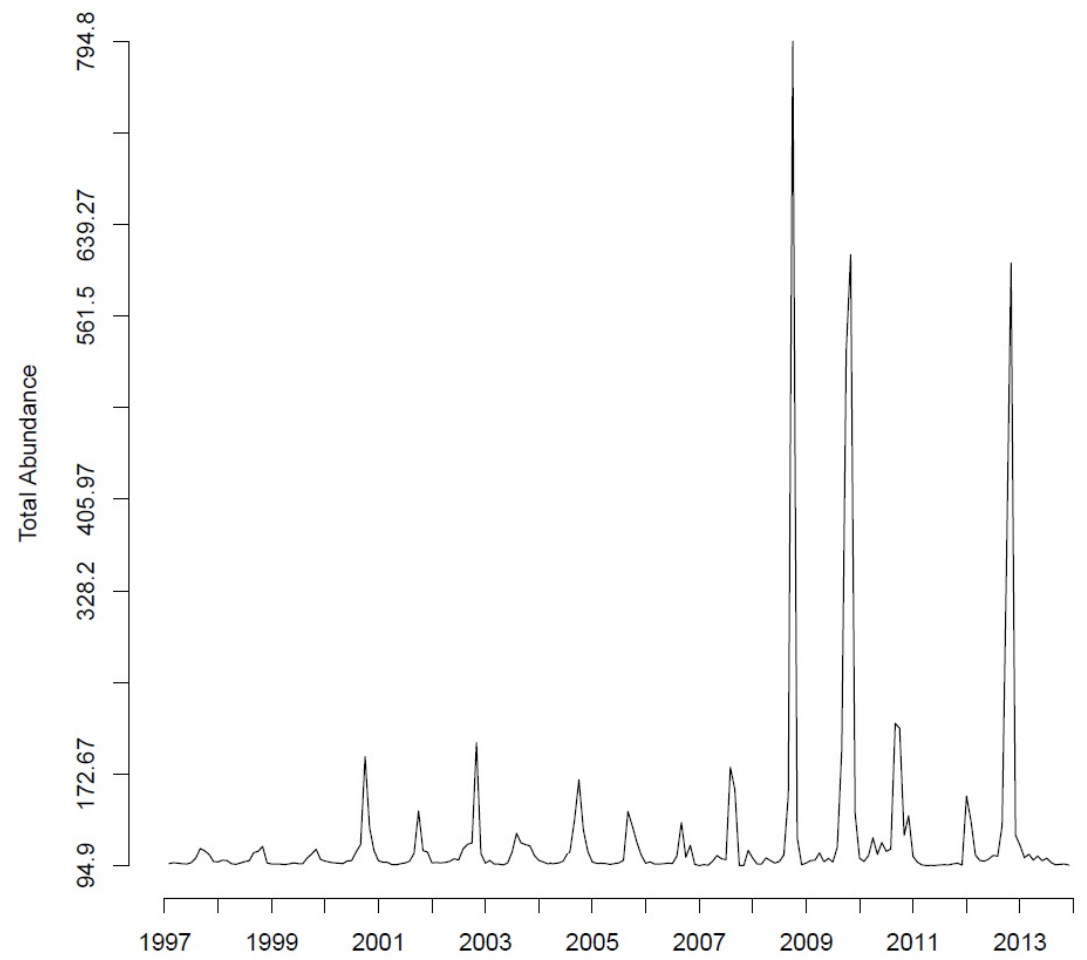



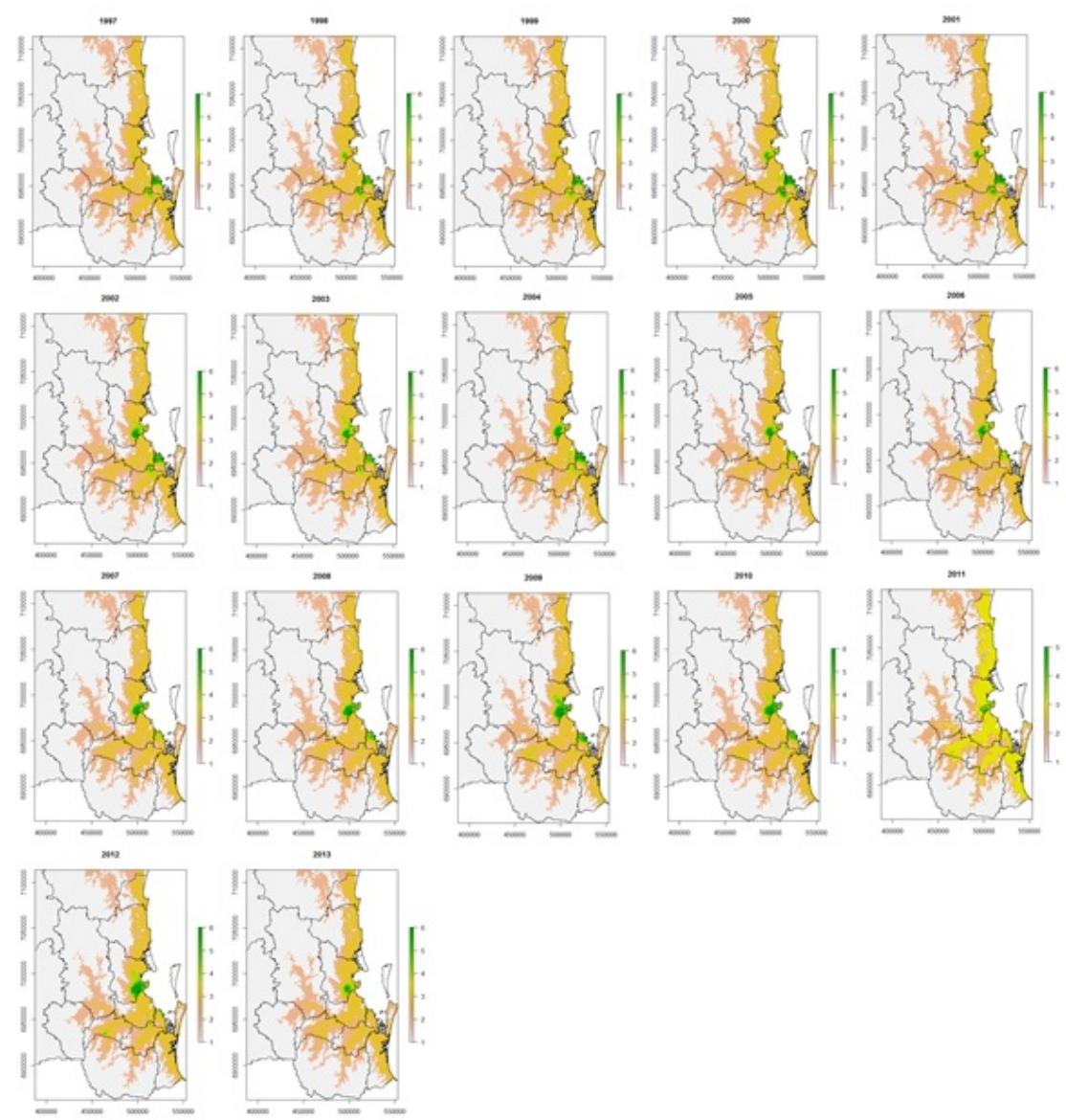